\title{
Fiber Lasers, a Future Technology for Lasers in Space
}

\author{
John M. McMahon \\ Naval Research Laboratory \\ Code 5601, Washington, DC 20375 \\ (202) 767-9322 \\ mcmahon@ccf.nrl.navy.mil
}

\begin{abstract}
The constraints of operation in space have largely precluded the use of conventional solid-state laser systems for applications including remote sensing, communication relays and active laser radars. A new technology, fiber lasers, may offer all of the needed features at an affordable price. An appealing aspect of the fiber laser is that it does not need a rigid optical bench. Only the output end of the fiber need be held in rigid reference to the optical tracking system. Design, fabrication and testing of the laser resonator is generally the most expensive and longest lead part of the effort for conventional solid-state lasers. Advances in Fiber Optic technology and devices mean that the "fiber laser" need not be a simple device but may be a complex system employing sophisticated technology such as wavelength selective Bragg reflectors and nonlinear optical frequency shifters. Three companies have recently obtained single-mode outputs of 3540 watts single mode at $1.03-1.1$ microns
\end{abstract}

\section{TABLE OF CONTENTS}

\section{INTRODUCTION \\ 2. FIBER LASER CONCEPTS \\ 3. DOUBLE CLAD FIBER LASERS \\ 4. YTTERBIUM LASERS \\ 5. FIBER LASER FREQUENCY SHIFTERS \\ 6. ULTRA-HIGH POWER FIBER LASERS \\ 7. CONCLUSIONS \\ 8. REFERENCES \\ 9. BIOGRAPHY}

\section{INTRODUCTION}

The space environment places constraints on lasers and associated optical systems that are different from those in terrestrial applications. These constraints affect the types of lasers that may be suitable in space, creating requirements for: (1) long-lived and reliable unattended operation, (2) relatively efficient laser operation, and (3) laser operation formats that are suitable for the missions being contemplated.

The first of these requirements was the impetus for much of the work in developing laser diode pumping rather than flashlamp or arc lamp pumping for solid state lasers. With a lamp-pumped system, a lifetime exceeding 10 million pulses or -1000 hours for lamp operation is not very practical. With a diode-pumping system, lifetimes of 10 billion pulses and tens of thousands of hours are reasonable. Because desirable lifetimes for spacecraft systems are usually in the range of 5 to 7 years of operation, i.e. 43,830 to 61,360 hours, the diodepumped technology has a chance while lamp pumped systems have none.

The need for a reasonably efficient laser derives from the extremely limited power and thermal cooling available on spacecraft. For conventional solid-state lasers, the need for high efficiency is somewhat orthogonal to the need for high reliability. This is because the efficiency with which one will extract stored laser energy scales directly with how heavily the laser is loaded, that is, as a function of the energy or power density compared to the saturation values. For good extraction, one generally wants an energy or power density of three times the saturation energy or power density. For neodymium-doped yttrium aluminum garnet (Nd:YAG), a common solid-state laser material, this prescription would require an average energy density of $-1.2 \mathrm{~J} / \mathrm{cm}^{2}$ or 6,000 watts $/ \mathrm{cm}^{2}$. As the peak values of energy or power density will almost always be 1.5 to 2 times higher, parts of the optics will be exposed to more than 2 to $2.5 \mathrm{~J} / \mathrm{cm}^{2}$ and/or 10,000 watts $/ \mathrm{cm}^{2}$. At such intensities optical damage may occur, which is the second leading life limiter for conventional laser systems. This may not be a failure of the laser rod itself, but of other components in the laser cavity. Laser materials are rather robust; electro-optic modulators and multilayer dielectric mirrors or other coatings on optical surfaces are somewhat more fragile. In trying to improve efficiency, it is very easy to decrease reliability.

The laser formats, which one may desire of a spacegoing laser, will depend on the application. For a communications system, one may really desire a true continuous operation with an external modulator to turn the laser on and off at the desired modulation rate. In fact, the only time when one would want a pulse format with a sequence of short time duration pulses would be when one is trying to illuminate another object in space and use the time of flight to deconvolve the third dimension, object depth, from the twodimensional scene. (This is not the only way to do this of course. Frequency chirping the laser during the pulse, entirely analogously to what is done with RF radar, has also been demonstrated with lasers.) Short pulses are not required to reduce motion blurring. The object would have to be travelling rather rapidly for this to be a major consideration as a $10 \mathrm{~cm}$ blurring for a 10 nanosecond pulse corresponds to $10^{9} \mathrm{~cm} / \mathrm{sec}, 1 / 30$ of the speed of light. When the Navy and DARPA were experimenting some years ago with submarine laser communications, the reason for the chosen pulse format was an issue of having sufficient energy in each message bit to penetrate deep into the ccean. 
A relatively new technology, diode-pumped fiber lasers, which has been developed for the very different purpose of fiber-optic communications, seems to be well matched to the requirements for long-lived laser operation in space. It is also well matched in what it can do well relative to space mission requirements.

\section{FIBER LASER CONCEPTS}

Before reviewing recent results that suggest that laser diodepumped fiber laser technology has come of age, I will briefly review some of the key concepts and nomenclature used in this field. A fiber-optic waveguide is the optical frequency analog of a dielectric RF waveguide. The waveguide can be single- or multi-mode and of various materials and construction, such as glass or plastic. Generally, for fiber optical communications over any reasonable distance, a single-mode glass fiber waveguide is preferred. It consists of a core, typically two to three times the optical wavelength in diameter, surrounded by a somewhat thicker cladding glass layer of slightly lower index of refraction than the core. In the "typical" low-loss silica fiber, which has a loss of $\sim 0.15$ $\mathrm{dB} / \mathrm{km}$ for wavelengths of 1.3 to 1.55 microns, the core is fused silica, $\mathrm{SiO}_{2}$, with a small amount of germanium added. This addition slightly increases the index, which is useful for the waveguide role. A polymer sheath, as well as various environmental and strengthening layers, depending on the application, then surrounds the core-cladding region.

The cladding acts as a nonabsorbing medium in which the light field in the core can die down to negligibly small levels before coupling to the outside world.

If the cladding is insufficiently thick or absent, light can leak out of the fiber by an evanescent wave coupling phenomena. In one popular type of fiber-optic coupler, the cladding layer is removed from one fiber and the core of another fiber is placed alongside it. By adjusting the length in close contact, the splitting ratio can be adjusted between the two fibers to be anything between 0 and $100 \%$. The main function of the layers farther out from the core is to prevent any direct contact between the outside environment and the core.

The first fiber lasers used single-mode fiber, but with the core also doped with rare earth ions such as neodymium, or erbium. This laser could give very reasonable laser, but the pump laser was not so reasonable. To pump a single-mode fiber required a single-mode pump laser, as a multimode beam could not propagate efficiently down the fiber. What was immediately interesting about these lasers was that continuous output operation was readily achieved for a variety of lasing ions of both three- and four-level transitions. Usually, it is difficult to get three-level transitions to operate efficiently but this appeared to be an exception, for a fairly simple reason. In a single-mode fiber laser the pump power is confined to a region -3 microns in diameter, i.e., an area of $\sim 7 \times 10^{-8} \mathrm{~cm}^{2}$. 1 watt of pump power would be intensified to $1.4 \times 10^{7}$ watts $/ \mathrm{cm}^{2}$, on average in the waveguide. This is very strong pumping compared to typical lamp-pumped lasers at $1-2 \times 10^{3}$ watts $/ \mathrm{cm}^{2}$ and transverse diode pump lasers that operate at even lower pumping levels. It is not surprising that laser operation well over the three-level threshold was seen, perhaps for the first time, for erbium, as well as other rare earths. As a simple numerical example, for a laser transition similar to neodymium in many glasses with an energy gap from the ground state of $9400 \mathrm{~cm}^{-1}$ and a fluorescence lifetime of 300 microseconds, a pump intensity of $1.4 \times 10^{7}$ watts $/ \mathrm{cm}^{2}$ could maintain a population of $-2.2 \times 10^{22}$ ions $/ \mathrm{cm}^{2}$ in the upper laser level by counterbalancing fluorescent decay. This is equivalent to $100 \%$ of the ions in 15 meters of fiber doped at $1 \mathrm{wt}^{2} \% \mathrm{Nd}^{3+}$. Under conditions of such strong pumping, there may be strong excitation of higher order processes in the laser ions, such as upconversion and excited state absorption. This may be a desirable result for upconversion-enhanced lasers, or it may represent a loss of population from the desired process. It can also lead to damage to the fiber from high pump or generated intensities. This region of operation proved to be appealing to enter because of the wealth of new physics but was also very expensive because of the single-mode pump laser requirement.

\section{DOUble Clad Fiber LASERS}

A clever technique for replacing expensive single-mode pumps with less expensive multi-mode pumps was reported by Po et al. [1] In this case, the single-mode fiber core is placed asymmetrically into a multi-mode fiber core. (Too much symmetry is bad, as most rays would never intersect the small single-mode region.) In principle, because there is no absorption in the multi-mode cladding, all the pump light will be absorbed in the single-mode core, and similar efficiency to that in the single-mode pumping case should be seen, but now with an inexpensive multi-mode pump. In practice, the results are not quite as good because fabrication of the more complex double core fiber is not yet as mature as single core fiber. There is typically a loss at the interfaces between the two cores of $\sim 1 \mathrm{~dB} /$ meter for the commercially available double clad fibers. This should be reduced with time and experience, but even with such additional losses, very impressive performance has recently been reported by groups at Polaroid [2], Lucent Technology [3] and at SDL [4], most notably with diode-pumped ytterbium doped fiber lasers. Single mode output powers have been obtained in the range of $35-40$ watts continuous, with slope pumping efficiencies of 60 to $65 \%$ when pumped in the $910 \mathrm{~nm}$ spectral region with multi-mode laser arrays. Performance with other rare earth ions such as neodymium or erbium has generally been much more modest but still at the 3-10 watt level. The best fiber laser output to date exceeds that of the highest direct single-mode diode laser outputs by a factor of five to ten. This is quite impressive since all fiber laser results have been over the past four years, whilehigh power diode development has been ongoing for more than a decade. The highest power DFB (distributed feedback) diode laser oscillators commercially available from SDL Inc. produce $\sim 600$ milliwatts of power. Slightly more power, $\sim 1000$ milliwatts, is available from commercial SDL Inc. 
master-oscillator-power-amplifier (MOPA) structures. Singular devices from MIT/LL and a NRL-SDL collaboration achieved higher powers of up to 5 watts at 980 $\mathrm{nm}$ and $850 \mathrm{~nm}$, respectively, but these have not been generally available.

\section{YTTERBIUM LASERS}

Ytterbium is far from the best known laser ion. It lases in glass in bulk lasers in relative obscurity between $\sim 1.0$ and 1.1 microns. The main reason for its early obscurity is also the reason for its current fame and glory, the peculiar nature of the ytterbium ionic energy levels. What is peculiar is that it is a very simple diagram with only two levels, the ${ }^{2} F_{5 / 2}$ upper laser level and the ${ }^{2} F_{7 n}$ lower laser level. The only reason why laser action is possible for this ion in crystals and glasses is that the ytterbium energy states are split into $2 J+I$ Stark sub-levels by the atomic fields of the host materials. The five sublevels of the upper state and the seven levels of the lower state are split quite broadly, by 1 to $\sim 1.5 k T$ at room temperature. This means that one can maintain a population inversion between the lower-lying components of the upper level and the higher-lying components of the lower level. The near co-incidence of pump and lasing levels also leads to very little waste heat intrinsic to optical pumping. For Ytterbium, the quantum defect is $\sim 0.08$, much smaller than for neodymium where the quantum defect is -0.28 .

The main reason why ytterbium was avoided in the era of lamp-pumped solid-state lasers is that with only one narrow pump absorption into which to pump, the overlap with the spectrum of a quasi-blackbody source was not good. As a consequence, the pumping efficiency was low. The only efficient pump scheme was to co-dope the laser with neodymium [5] and make use of the broad and numerous neodymium absorption bands. Unfortunately, this then also results in the quantum defect for neodymium. A second reason was that the stimulated emission cross-section, or gain coefficient per ion, was - four times smaller for ytterbium than neodymium. For pulsed flashlamp-pumped operation, a four times higher energy density was necessary to extract the same fractional stored energy density.

For a diode-pumped fiber laser, these properties of ytterbium in glass are advantages rather than problems. The narrow absorption is still considerably broader than the spectrum of the pump diodes, so there is a -3 -fold advantage in reduced heating. For quasi-cw operation, the parameter of interest in scaling laser performance is the power saturation fluence, which is proportional to the product of the induced emission cross-section and the fluorescence lifetime; this is essentially the same for either neodymium or ytterbium in most glasses. The one disadvantage of ytterbium for fiber-optic communications, the emission wavelength of one micron, versus the desired 1.3 and/or 1.55 microns is also essentially common to both ytterbium and neodymium. However, the smaller quantum defect and longer fluorescence lifetime are advantages. The equal power saturation intensity and higher single-pulse saturation energy density are more favorable for a high data rate analog communication system (greater fidelity) and at least equal for digital communications. For long-haul fiber telecommunications, frequency shifting of either laser is required. Some very attractive technology has been developed here recently and will be covered in the next section, but it is worth noting that, for a free space communications system using silicon PIN or avalanche detectors, the fiber laser output at one micron is more attractive than the longer wavelengths preferred in fibers because of reduced diffraction spreading of the beam.

A ytterbium fiber laser that produces 10 watts of single mode output when operated as an oscillator can also be operated as an amplifier with more than $32 \mathrm{~dB}$ of small signal gain for a few milliwatt laser diode source. It can produce about 10 watts of modulated output with a sufficient gain bandwidth that frequency multiplexing can be used to push data rates up into the $\sim 5$ tolo Gbit/sec range. This is a very attractive prospect for a communications system because not only does it appear that the key components already have been demonstrated, they have been developed and will be further reduced to reliable, cheap off-the-shelf status by the phone company and others for local area networks (LANs) and for cable TV signal distribution. An additional benefit of a master-oscillator-power-amplifier combination is that the fiber amplifier can be operated from a DC power supply and only the low power oscillator modulated at a high repetition rate. Up to $-1 \mathrm{Gbit} / \mathrm{sec}$, this is probably the simplest approach. For higher data rates, the low power of the oscillator allows the use of a modulator between the oscillator and the amplifier. In fact, at this point in the device apparatus can be identical to that used in fiter-optic systems. In addition to high data-rate digital equipment, .travellingwave lithium niobate modulators have been developed recently for a number of analog applications in radar and electronic warfare in which a RF signal is coherently superimposed on an optical carrier. These modulators have a flat frequency response out to $50 \mathrm{GHz}$ with a 3 to 5 modulation voltages [6]

An additional benefit is the relatively high overall efficiency of these lasers. In a recent paper from Lucent Technologies [7], an ytterbium fiber laser with outputs of 20.4 watts at $1101 \mathrm{~nm}$ required only 35 watts of multi-mode diode pump power. The efficiency of the diode array is not given but typical arrays at this pump wavelength have efficiencies of 35 to $45 \%$. Thus the input power from the satellite bus would be less than 100 watts and most of the waste heat will be in the pump array and its power supply which can be located wherever convenient in the satellite and not in the fiber laser. For a relay satellite in synchronous orbit, this would appear to be close to a complete system solution.

For low-altitude satellites, a laser wavelength of 1 to 1.1 microns may not be desirable from the standpoint of possible eye safety issues. This is not a foregone conclusion because: (1) the hazards to astronauts and cosmonauts from a direct exposure are not large except at very short range, and (2) such situations could presumably be avoided by careful 
mission planning, but ( 3 ) there could be a hazard situation for terrestrial observers high magnification telescopes. This last situation is hard to define quantitatively but prudence might dictate use of a nominally eyesafe 1.35 to 1.55 micron wavelength. While this wavelength would eliminate retinal damage concerns, it would not eliminate the possibility of all eye damage to amateur astronomers. If sufficient flux were collected, damage to the front surfaces (cornea and lens) of the eye would still be possible. Total elimination of laser risk to eyes cannot be done only at the source; for total safety, one would want to have an element in the optical train of instruments with high magnification that blocked the laser wavelengths. This blockage would be relatively easy for the 1.3 to 1.55 micron region without interfering with any other uses of the instruments.

\section{FIBER LASER FreQuenCy SHIFTERS}

Notable progress has been made in fiber laser frequency shifters over the past several years. The generic difficulty in the past has been that nonlinear processes of frequency conversion which have the desirable property of preserving the amplitude and phase of the initial wave (usually as a convolution function rather than directly) only could be made to work at high intensity. Linear processes, such as laser pumped lasers, could be made to work at low intensities, but they integrate the pump power and hence destroy the information stream. In two different areas, quasi-phase matched waveguide materials and fiber Raman converters, researchers have demonstrated new nonlinear devices which operate at low intensity but are non-linear converters. There are no direct bulk optics analogs to these devices, although research is underway to utilize optical or fusion bonding to increase the aperture area, and hence power handling capability, of the quasi-phase matched waveguides.

A quasi-phase matched structure is one in which the axes directions in a birefringent crystal are inverted with a periodicity corresponding to the mutual coherence length of the pump and signal waves in the nonlinear process. This periodicity is typically 30 microns, which corresponds to very thin crystal slices if fabricated conventionally. With lithium niobate crystals, there is another approach to generating such regions, by electrically poling the crystal. [8] This approach has a limitation in crystal transverse dimension of $\sim 1 \mathrm{~mm}$ in that very high electric field strengths are needed for poling, which suggested that it could best be used in the form of a rectangular waveguide. There is no real limit on the interaction length for the waveguide, rather quickly, researchers were able to demonstrate mid-IR continuously operated optical parametric oscillators at an average power level of 3.5 watts output in the signal wave for a 10 watt pump [9], [10]. Because of this success and the limited transparency and damage-free regimes for 1 lithium niobate, considerable effort is ongoing to find other materials that might be polable but more broadly useful, as well as to produce the equivalent quasi-phase matched structure by synthesizing stacks of crystal laminae [11]. To be useful, this latter approach needs to develop techniques for producing very low scatter surfaces that result in a composite with internal losses similar to a monolithic crystal.

Fiber optics is also a waveguide structure, although of an amorphous material, glass. A powerful beam sent down a long length of waveguide will generate forward and backward travelling waves at shifted frequencies by stimulated Brillouin scattering. Suppressing this process was, in fact, one of the hurdles that fiber-optic communication had to overcome to propagate coherent signals over long distances. Suppression is achieved by broadening the bandwidth of the laser such that the coherence length becomes shorter than the physical length of the fiber. When a source is modulated at a high frequency as in a fiber optic system, such broadening is a usually beneficial side consequence of the modulation process. The Brillouinshifted wave is not always undesirable. It is a phase conjugate wave to the input wave, so the full range of optical phase conjugation phenomena is potentially possible.

A process that can compete with Brillouin scattering in glasses, as well as other solids and gases, is stimulated Raman scattering. In a waveguide, generation of radiation other than the longer wavelength Stokes shifted component is automatically suppressed because the anti-Stokes gain can only be non-zero at an-off-axis angle. The frequency shift for a Raman process will depend on the bonding energies in the typical material. For germanium silicate glass, the largest gain is at a shift of $\sim 440 \mathrm{~cm}^{-1}$.

To promote Raman over Brillouin, feedback at the Ramanshifted frequency can be added by impressing so-called Bragg gratings on the fiber. To make these devices, a UV source such as a $\mathrm{KrF}$ excimer laser is used to produce a Fresnel diffraction pattern with the fringe transverse to the fiber and a periodicity of half the desired wavelength in the fiber. Absorption by the Germanium in the glass in the high intensity regions will cause a small permanent local index of refraction change $\left(\sim 4 \times 10^{-4}\right)$ [12]. The Bragg grating structure can easily be made to extend over centimeters of fiber length and can give very strong and highly peaked reflection about the design wavelength.

Stentz [7] gives an elegant example of the use of multiple super-imposed Bragg grating Raman resonators to shift the frequency of an Ytterbium fiber laser from 1100 to $1472 \mathrm{~nm}$ in a five-step Raman process. In such an arrangement, each successive Stokes shifted component is the pump for the next process. Since only the final (fifth) component is desired as output, the Bragg gratings for all other wavelengths are designed to reflect $\sim 100 \%$ of the light at these wavelengths. In such an arrangement, the first observed output is at the final wavelength. The threshold reported by Stentz was less than two watts of ytterbium laser pump power.As Raman gains in solids are typically $10^{-10}$ per watt, the advantage of confining the beam in a fiber and, in effect stretching the beam waist from tens of microns to tens of meters is apparent. Figure 1 shows schematically how the fiber gratings were arranged. 


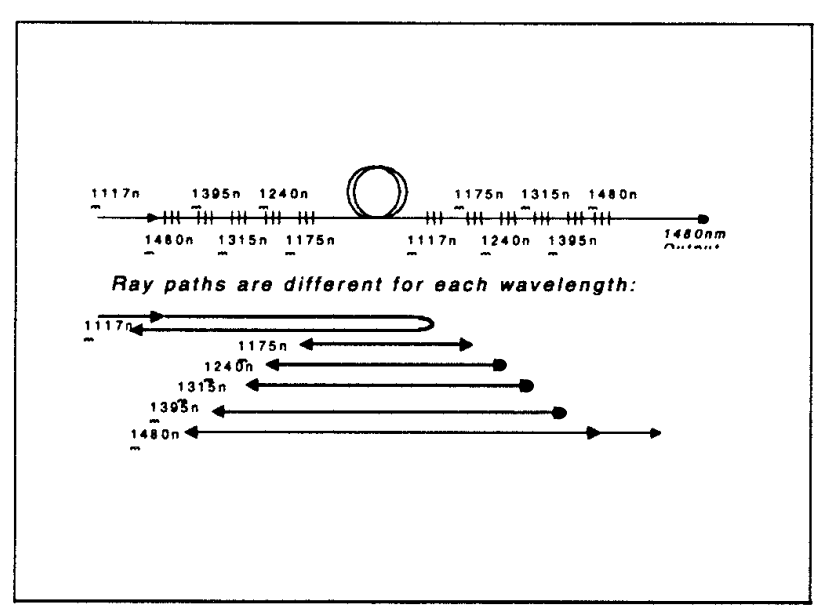

Figure 1.Geometry of Cascaded Raman Oscillator. The Fiber Bragg gratings define five Raman oscillators nested within each other such that the nth Stokes radiation is the pump for the $(n+1)$ th order Stokes radiation. All gratings also have nominal $100 \%$ reflectivity, except for the initial grating which admits the 1.03 micron pump, and the final grating which allows $\sim 50 \%$ of the 1.48 micron $5^{\text {th }}$ Stokes radiation to pass. All Fiber optics used in this laser is ordinary single mode-silicate, it does not require double-clad fiber

It is remarkable that the efficiency of this five-step nonlinear conversion process is $\sim 47 \%$, compared to a possible $67 \%$ based on the quantum defect, but there are very few losses in this system. The fiber is very low loss, the gratings are low loss, and there are no other components. An issue with strongly resonated systems such as this one is that the modulator has to be at the output end and bear all of the power. For a quasi-cw free space system this is not too serious an issue because the modulator aperture can be chosen to be wherever in the range between 3 microns in diameter and $30 \mathrm{~cm}$ in diameter, a comfortable trade between modulation speed, cost and safety is found.

\section{Ultra-High POWER Fiber LASERS}

At present, the power of electrically excited lasers in space is limited by an approximate $1 \mathrm{~kW}$ maximum available electrical power because of satellite electrical and thermal considerations. Potentially, a $\sim 200$ Watt ytterbium fiber laser could be operated, based on a $20 \%$ overall efficiency. One notably attractive feature of fiber lasers is that the diode laser pump system will operate at reasonable and moderate voltages. A laser diode pump bar is a reasonable approximation to a 1 to $1.5 \mathrm{ohm}$ device. At 20 watts output, with an efficiency of $45 \%$, the electrical demands would be on the order of 8 amperes and 5.5 volts. A five bar series stack, a very common configuration, would want a supply voltage of 28 volts DC. By various series-parallel arrangements spacecraft bus voltages could be readily matched with no additional power conditioning stages, unlike lamp pumped solid-state lasers. The laser architecture is an interesting issue at this power level. While powers of 35-40 watts have been reported from single fibers [2], [3], [4], these levels are really pump and not fiber limited. Work done at NRL on off-axis coupling of diode arrays into fiber lasers could allow efficient addition of a rather artitrary number of additional pump modules [13] into a double-clad fiber. Only a few effects would provide a physical upper limit on how high a power could be generated from a single fiber: (1) at some point, nonlinear gains will be high enough that Brillouin scattering cannot be avoided; (2) the intensity at which the beam no longer has gain in a fiber amplifier will be numerically equal to the saturation intensity times the ratio of gain per unit length to loss per unit length. The efficiency of any lossy amplifier thus becomes worse as the power density becomes high; (3) heating of the fiber core region will make the laser more three-level like by increasing the ground state population; and (4) damage will limit the power at some point, but by selecting fiber sections, this should not be a fundamental problem. Experiments are under way at McDonnell-Douglas to experimentally determine where these upper bounds are located. At the 1997 DLTR meeting, it was reported that powers of up to 100 Watts in very long 100 microsecond pulses had been propagated down 40 meter lengths of $\mathrm{Nd}$-doped double core fiber before the onset of stimulated Brillouin scattering (SBS) [14].

This research may indicate that as much as 100 watts per fiber may be achievable, although amplification efficiency at this power level is not yet established. Certainly, the results from a number of organizations indicate that the safe limit is at least 35 to 40 watts per fiber. Our notional 200 watt laser could be rendered fully coherent by phase controlling somewhere between two and six laser beams. In most important aspects, this problem is no more difficult than phase controlling an array of a like numbers of diode lasers. The payoff, at 100 times higher power per element for fiber lasers than diode lasers, is considerable. Research is currently underway, sponsored by the Air Force, to evaluate this possibility.

\section{CONCLUSIONS}

In a very short time, fiber lasers have been developed for fiber optic telecommunications which also exceed the entrance requirements for direct optical communications. Continued development for digital fiber-optic applications and for analog applications such as cable television will make the basic devices, as well as ancillary signal processing and conditioning equipment even more affordable. The system properties and impacts of this technology in space appear more reasonable than those of the conventional solid-state lasers they may replace. This may well one of a few places where commercial-off-the-shelf components can be rapidly and inexpensively tailored to meet particular space mission requirements. At present, fiber laser powers of the order of 40 watts would require of the order of 200 watts of power from the spacecraft power bus, a reasonable requirement. In the more distant future when one would expect higher power 
bus capabilities on satellites, extension of fiber laser technology by using phased arrays of fiber lasers is likely to be the technology at the forefront of overall efficiency and yield the highest total laser power in a coherent beam.

\section{REFERENCES}

[1] H. Po, J.D. Cao, B.M. Laliberte, R.A. Minns, R.F. Robinson, B.H.Rockney, R.R. Tricca, and Y.H. Zhang, Electronics Letters 29,p.1500 (1993)

[2] P. Gavrilovic, $10^{\text {th }}$ Annual Diode Laser Technology Review, Albuquerque, NM (June 1997)

[3] D. Inniss, et.al. submitted to postdeadline session of CLEO/QELS, Baltimore, MD(1997)

[4] S. Grubb, 10 th Annual Diode Laser Technology Review, Albuquerque, NM (June 1997)

[5] A. D. Pearson and S.P.S. Porto, "Nonradiative energy exchange and oscillation in $\mathrm{Yb}^{3+}-\mathrm{Nd}^{3+}$ - doped borate glass," Appl. Phys. Lett., vol. 4, pp. 202-204, June 1964.

[6] G. K. Gopalakrishnan, W. K. Burns, R.W. McElhanon, C. H. Bulmer and A. S. Greenblatt, "Performance and Modeling of Broadband LiNbO3 Traveling Wave Optical Intensity Modulators", Journal of Lightwave Technology, Vol.12, No.10, pp 1807-1819, Oct. 1994.

[7] A. Stentz, $10^{\text {th }}$ Annual Diode Laser Technology Review, Albuquerque, NM (June 1997)

[8] L.E. Myers, R. C. Eckhardt, M. M. Fejer, R. L. Byer and W. R. Bosenberg, "Multigrating quasi-phase-matched optical parametric oscillator in periodically poled $\mathrm{LiNbO}_{3}, " O p t$. Lett. 21, 591-593 (1996)

[9] W. R. Bosenberg, A. Drobshoff, J. I. Alexander, L. E. Myers AND R. L. Byer, "Continuous-wave singly resonant optical parametric oscillator based on periodically poled LiNbO,", Opt. Lett. 21, pp. 713-715 (1996)

[10] W. R. Bosenberg, A. Drobshoff, J. I. Alexander, L. E. Myers AND R. L. Byer, " 93\% pump depletion, 3.5-W continuous-wave singly resonant optical parametric oscillator ", Opt. Lett. 21, pp. 713-715 (1996)

[11] H. Meissner, Onyx Optics Inc., 1997 Phase I SBIR for the Naval Air Systems Command, U.S. Navy.

[12] J. D. Prohaska, E. Snitzer and J. Winthrop, Appl. Optics 33, p.3896 (1994)

[13] L. Goldberg, $10^{\text {th }}$ Annual Diode Laser Technology Review, Albuquerque, NM (June 1997)
[14] C. L. Balestra, J. H. Hollister, J. L. Levy, B. C. Johnson, R. R, Rice and M .S. Zediker, I ${ }^{\text {th }}$ Annual Diode Laser Technology Review, Albuquerque, NM (June 1997)

\section{BIOGRAPHY}

John M. McMahon. Born in 1941 in St. Paul, Minnesota. BS in Physics from Boston College in 1963. Master of Arts in Physics from Dartmouth College in 1965. Employed at NRL as a summer student in 1961, 1962,1963 and 1964 in Optics and Radiation Divisions. Areas of concentration were nuclear weapons effects, radiation transport, and high power plasma physics devices. Became a permanent NRL employee in 1965.From 1965 to 1982 concentrated on the development of high power solid-state lasers for ARPA (DOD), Defense Nuclear Agency, and Department of Energy applications. Developed first successful high peak power disc laser amplifiers. Developed a laser system at NRL suitable for well-characterized laser plasma experiments in aid of activities in inertial confinement fusion (laser fusion), laser generation of $\mathrm{x}$-rays and nonlinear phenomena in laser produced plasmas. Published over 100 articles, papers, reports and talks in both laser and laser plasma areas. In 1976 published a then-controversial position advocating phosphate laser glasses as the most advantageous new laser materials for the large lasers such as NOVA at LLNL then under construction in the US. This position became the main line direction in 1979 and he received the Meritorious Civilian Service Award from the DOD for this effort.

From 1991 to the present he has been employed in NRL's Optical Sciences Division; from 1981 to 1985 as Associate Superintendent of the Division and from 1986 to the present as Chief Scientist and Acting Associate Superintendent of the Optical Sciences Division. In these roles he has been in an oversight position over a group of several hundred scientists and engineers involved in a broad variety of disciplines in electro-optics. Particular concentration exists in all aspects of fiber optics including new materials, such as fluoride and calcogenide fibers, fiber optical sensors, fiber amplifiers, and optical control of phased array structures. The infrared program has been one of the nuclei of the DOD effort to develop affordable focal plane array sensors with both Mercury Cadmium Telluride and Indium Antimonide Sensors. The program has also strongly emphasized research on development of sensors employing these detectors for both space and terrestrial applications including surveillance and reconnaissance as well as the high priority missile threat warning receivers. The laser related activity has concentrated on developing lasers with capabilities in the infrared bands where the atmosphere is transparent and also in exploiting nonlinear optical effects for laser beam correction and combination and image enhancement and processing.

He has aiso been involved for some years as advisor to the DOD in areas of export control policy and has participated in a number of international negotiations. (Perhaps the high 
point in this area was when he was Acting Ambassador and President of COCOM for one session in 1993.) He chaired the Technical Working Group on Sensors for 1992-1994, which involved DOD, State Energy and Commerce participants.

He has also been active since 1990 as the Navy Chairperson for the Electro-Optics and Lasers Areas in Project Reliance and the current TARA Review Process. He was the TriService Chairperson for all of Electro-Optics for the period of 1993-1996. He has also served as a consultant or advisor to the Army Science Board, the Air Force Scientific Advisory Board, the Defense Special Weapons Agency, as well as the Department of Military Affairs of the Department of Energy. 